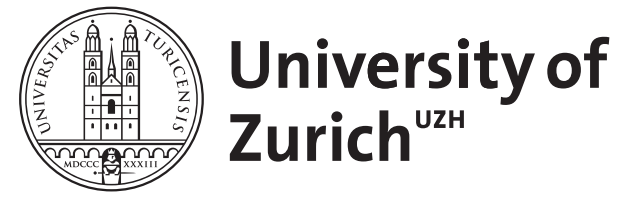

Year: 2016
Zurich Open Repository and Archive

University of Zurich

University Library

Strickhofstrasse 39

$\mathrm{CH}-8057$ Zurich

www.zora.uzh.ch

\title{
Mild heisst nicht banal
}

\author{
Rosemann, Thomas
}

DOI: https://doi.org/10.1024/1661-8157/a002372

Posted at the Zurich Open Repository and Archive, University of Zurich ZORA URL: https://doi.org/10.5167/uzh-130350

Journal Article

Accepted Version

Originally published at:

Rosemann, Thomas (2016). Mild heisst nicht banal. Praxis, 105(10):541.

DOI: https://doi.org/10.1024/1661-8157/a002372 
Der Hausarzt ist nach wie vor für die meisten Patienten der erste Ansprechpartner. Das gilt nicht nur für die heute so intensiv diskutierten chronischen Erkrankungen, sondern auch für akute Behandlungsanlässe, inklusive Verletzungen. Die weit überwiegende Zahl dieser Behandlungsanlässe kann der Hausarzt dabei auch abschliessend behandeln, das heisst sie bedürfen keiner Weiterverweisung. Wirklich verlässliche Zahlen gab es dazu aus der Schweiz bisher nicht, aber eine aktuelle Studie des Zürcher Instituts für Hausarztmedizin an der sich 90 Hausärzte beteiligten, belegte dies eindrucksvoll: fast 25.000 Konsultationen wurden erfasst, dabei erwähnten die Patienten 42.890 verschiedene Konsultationsgründe und Beschwerden. Auf Basis der Konsultationsgründe mussten nur 5,6\% weiterverwiesen werden (1). Eine eindrucksvolle Zahl, wie ich denke. Zahlen, die aber erneut auch die grosse Herausforderung des Hausarztes deutlich machen: zu erkennen in welchen Fällen ein „abwendbar gefährlicher Verlauf“ (so der mittlerweile etablierte Fachterminus) vorliegt und eine unmittelbare Reaktion oder gar Weitererweisung notwendig sind. Herausfordernd ist dies umso mehr bei akuten Ereignissen und Traumata. Schädel-Hirn-Traumata, insbesondere im Sinne leichter oder milder Gehirnerschütterungen zählen in unserer so aktiven und sportlichen Gesellschaft nicht selten zu diesen Konsultationsanlässen. In der Schweiz führen Gehirnerschütterungen pro Jahr zu über 20.000 Hospitalisationen pro Jahr, eine Zahl die ebenso überrascht wie beeindruckt. Reto Stocker und Claudio Letta schildern in dem sehr gut strukturierten Übersichtsartikel auf Seite ... in diesem Heft sehr anschaulich die Epidemiologie aber vor allem auch die klinische Problematik dieser Verletzungen. Und wie so oft in der Medizin zeigen sie sehr eindrucksvoll auf, das eine definitionsgemässe „milde“ Ausprägung nicht bedeutet, dass das zu Grunde liegende Krankheitsbild banal und vor allem folgenlos bleibt. Mich hat die Zahl von $15 \%$ überrascht, die angibt, wie viele Patienten auch noch nach einem Jahr und länger Beschwerden und Beeinträchtigungen erleben. Die Gefahr, dass Menschen dabei leichtfertig als Simulanten abgestempelt werden, weil scheinbar kein kausaler Zusammenhang mit der initial ja „milden“ Verletzung vorliegen könne, ist evident. Daher denke ich gibt der Artikel nicht nur wertvolle Hinweise für den Alltag sondern erzeugt zugleich eine Aufmerksamkeit für ein manchmal sicher unterschätztes, vermeintlich mildes Trauma.

(1) Referral rates in Swiss primary care with a special emphasis on reasons for encounter. Tandjung R, Hanhart A, Bärtschi F, Keller R, Steinhauer A, Rosemann T, Senn O. Swiss Med Wkly. 2015 Dec 28;145:w14244. 